\title{
A Study on Fault Diagnosis of Redundant SINS with Pulse Output WANG Yinan ${ }^{\mathrm{a}}$, REN Zijun ${ }^{\mathrm{b}}$, DONG Kaikai ${ }^{\mathrm{a}}$, CHEN kai ${ }^{\mathrm{a}}$, YAN Jie ${ }^{\mathrm{a}}$
}

School of Astronautics, Northwestern Polytechnical University, Xi'an 710072, PR China

aemail: chenkai@nwpu.edu.cn, bemail: renzj8888@163.com

Keywords: Redundant SINS; Fault Diagnosis; GLT; Pulse Quantization Output; Low Pass Filter

\begin{abstract}
The missile-based redundant SINS (strap-down inertial navigation system) output the measurement data by using pulse quantization. Pulse quantization can disorganize the errors in the original measurement signal, cause an instantaneous drift of 50 degree/hour, and thus brings about great difficulty to the process of fault diagnosis. Focusing on the problem that the pulse quantization results in difficulty to the fault diagnosis of the redundant SINS, this paper proposes a method that using a low pass filter reduces the quantization noise. Based on GLT (generalized likelihood test) method, one of the methods used for fault diagnosis of the redundant SINS and combined with a low pass filter, this paper studies the effects before and after the fault diagnosis comparatively and draws a conclusion that a low pass filter is helpful for improving the effect of quantitative fault diagnosis. The result of the simulation experiment shows that the effect of the fault diagnosis after improving is close to that before using pulse quantization, and that the effect can detect the order of 5 degree / hour accurately.
\end{abstract}

\section{Introduction}

With the development of strap-down inertial navigation technology, SINS has become one of the most important equipment in rockets. The reliability of SINS determines the reliability of the missile borne control system, and once the control system fails, a serious accident would happen. Therefore, it is of great necessity to improve the reliability of SINS, wherein using the redundant method [1-4] to improve the reliability of SINS is feasible. When a fault occurs, detecting and isolating the inertial sensor instantaneously would be another problem to study [5-11]. Based on the redundancy configuration of 6 sets of IMU, this paper discusses the fault diagnosis method, compares the effect of fault detection before and after quantization output, and accordingly solves the problem that the quantification bring about to fault diagnosis.

\section{The Model of SINS}

An IMU component (including a gyroscope and an accelerometer) is installed in each axis, i.e. $O x$ axis, $O y$ axis, and $\mathrm{Oz}$ axis for the conventional SINS. In order to improve the reliability of SINS, 6 sets of IMU components are installed by the approach of regular dodecahedron, wherein one axis is different from the other (Fig.1).

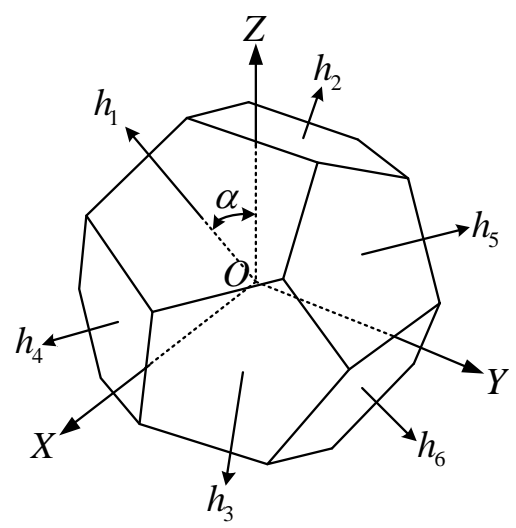

Fig 1. The configuration of the IMUs 


$$
Z=\left(I+H_{\text {sf }}\right)\left[\left(H_{n}+H_{\text {ma }}\right) X+b+\varepsilon\right]
$$

where, $Z$ is the measurement value of a gyroscope or an accelerometer, $Z \epsilon R^{N \times 1}, N$ is the number of the gyroscope or the accelerometer; $I$ is identity matrix; $H_{s f}$ is scale factor error matrix, and also diagonal matrix; $H_{n}$ is nominal mounting matrix, $H_{n} \in R^{N \times 3}$; the row vector is the space orientation of the corresponding sensor measuring axis; $H_{m a}$ is misalignment error matrix, $H_{m a} \epsilon R^{N \times 3} ; X$ represents inertial information of missile, i.e. three axis attitude angular velocity or three axis acceleration, $X \epsilon R^{3 \times 1} ; b$ is the bias of gyroscope of acceleration, $b \epsilon R^{N \times 1} ; \varepsilon$ is the noise of the sensor.

Nominal mounting matrix $H_{n}$ is:

$$
H_{n}=\left[\begin{array}{ccc}
0.52573 & 0 & 0.85065 \\
-0.52573 & 0 & 0.85065 \\
0.85065 & 0.52573 & 0 \\
0.85065 & -0.52573 & 0 \\
0 & 0.85065 & 0.52573 \\
0 & 0.85065 & -0.52573
\end{array}\right]
$$

\section{Analysis of Output Quantization}

Generally, the missile borne SINS outputs the quantitative measurements by using the form of pulse increment. The quantization error is introduced into the process of pulse quantization[12-13], as is shown in Fig.2:

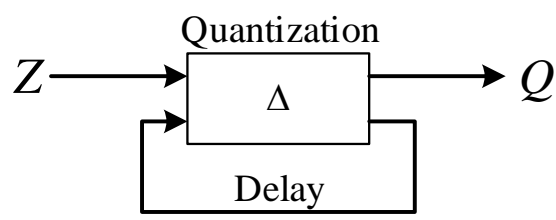

Fig 2. Quantization of the output

Quantization will enlarge the measurement error. Take gyroscope as an example, pulse equivalency is set to $1^{\prime \prime}\left(4.8 \times 10^{-6} \mathrm{rad}\right)$, sampling period $\Delta t=0.02 \mathrm{~s}$. When the rocket motor is small, the angular increment in the sampling period would be less than one pulse equivalent, so the output of the cycle would be 0 . As is shown in Fig.2, the angular increments (fractional) within the $\mathrm{k}$ period would be accumulated to the first $k+1$ cycle or to the $k+2$ cycle for outputting, and even to the $k+n$ cycle. The output of pulse equivalency is equal to pulse equivalency $1 " / \Delta t=50^{\circ} / \mathrm{h}$ in the sample period, which is equivalent to the instantaneous drift of $50 \%$ in the situation of low maneuver. The error of measurement is usually less than $1 \%$. That is to say, after quantization, the instantaneous error is likely to be more than 50 times, which would bring great difficulty to fault diagnosis.

\section{GLT Fault Diagnosis Method}

GLT algorithm is a method that using Potter algorithm constructs decoupling matrix $V$.

Define equivalence vector $p$ :

$p=V Z$

Define decision function $F_{D}$ :

$F_{D}=p^{T}\left(V V^{T}\right)^{-1} p$

If $F_{D}>T d$, then determine that a faults occurs; otherwise, no fault. $T d$ is the preset default threshold.

When a fault occurs, isolate the fault. The isolated decision function $F_{I}$ : 


$$
F_{I}^{j}=\frac{\left[p^{T}\left(V V^{T}\right)^{-1} v_{j}\right]^{2}}{v_{j}^{T}\left(V V^{T}\right)^{-1} v_{j}}
$$

wherein, $v_{j}$ is the column $\mathrm{j}$ of the matrix $V$.

Calculate $F_{I}{ }^{j}(j=1,2, \ldots m)$ respectively, and then find out the maximum one. For example, the maximum one is ${F_{I}}^{k}$, then the No. $k$ senor is considered to be a fault.

\section{Compasion of the Effect of Fault Diagnosis before and after Quantization}

Fault Diagnosis before Quantization:

When the errors in the inertial navigation output are not quantified, the noise term would be the largest, and the noise term plays a leading role. Therefore, in GLT detection method, the decision function $F_{D}$ is also close to $k \varepsilon^{T} \varepsilon$.

In 30 seconds, the step signal of $5 \% / h$ to the 1 gyro is injected. Then a step uplift of the fault detection decision function would appear in the 30 seconds, as is shown in Fig.3(a), which provides a condition for fault diagnosis. Under the default threshold $T d, F_{D}$ is detected continuously. At a certain time, if $F_{D}$ is beyond the threshold and maintains a period of time (for example, 5 sampling periods can effectively avoid the wild value point interference), the fault would be determined. Then, transfer to the separation step, immediately.

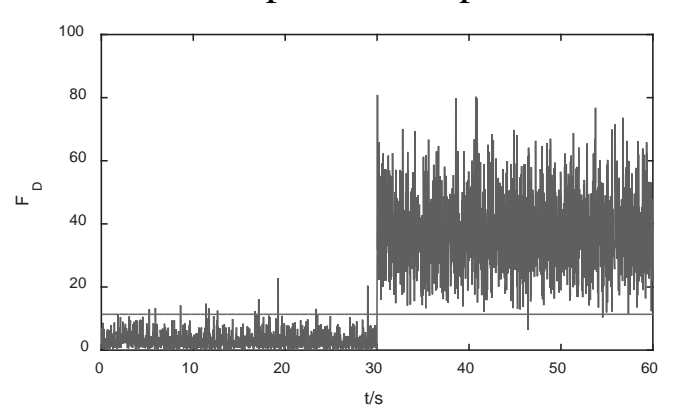

(a) Detection of fault;

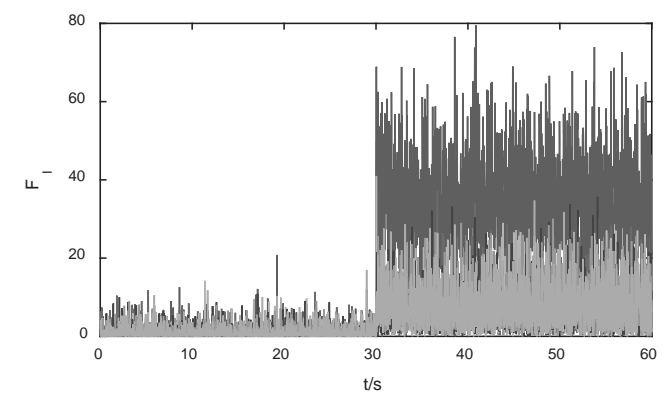

(b)Isolation of fault

Fig 3. The FDI result before quantization

Calculate respectively, the maximum one would be the fault sensor, as is shown in Fig 3(b). In order to reduce the error isolation rate, the average number of each sensor in a number of sampling periods can be calculated, and isolate the maximum one.

Fault Diagnosis after Quantization:

As has been analyzed previously, quantization will bring about error amplification. The error in the output $Q$ is irregular. Due to the quantization error, the decision function is enlarged many times in GLT detection method, and the default threshold is not applicable any more, as shown in Fig.4.

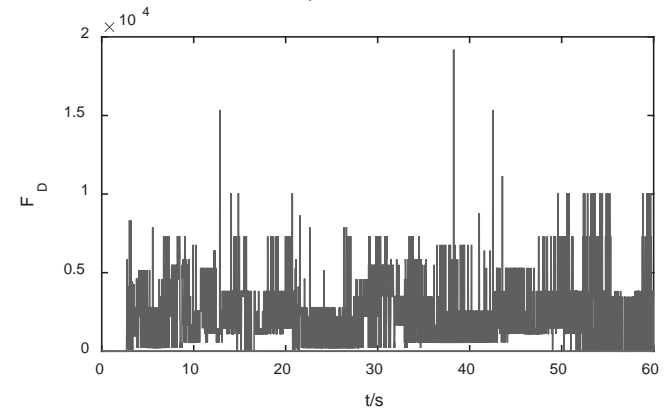

(a) Detection of fault;

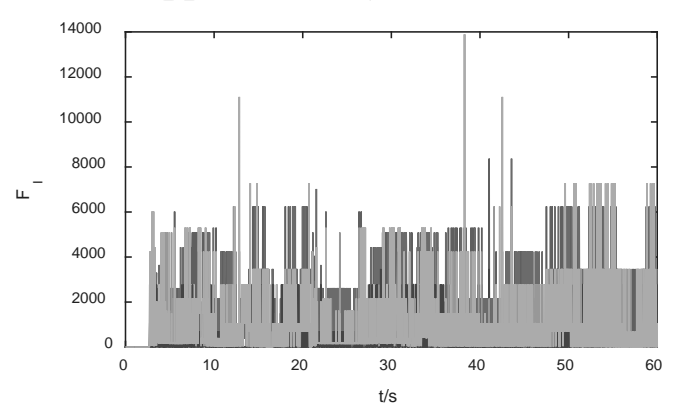

(b) Isolation of fault

Fig 4. FDI result after quantization

Similarly, the step type fault signal of $5 \% / h$ is injected into the 1 th gyro at the 30 th second. The fault detection decision function does not appear step uplift, but there is a discontinuous jump at the 30th second. Compare Figure 3 (a) and Figure 4 (a), we can clearly see that the effect changes fault diagnosis before and after quantitation. 
Based on the previous analysis, generating a pulse output requires an angular speed of $50 \% / h$, while the additional step fault of $5 \%$ would generate an additional pulse output in 10 sample cycles.

Through the simulation experiment, this paper finds: after quantitation, only when the amplitude of the step fault is 75 , can we obtain the effect of fault diagnosis that is closed to the effect before quantization, as shown in Figure 5.

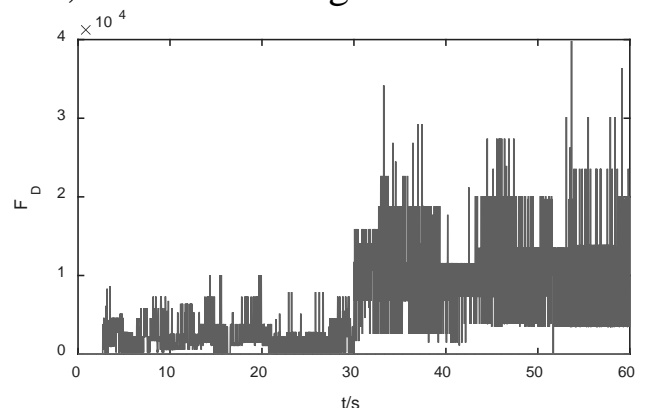

(a) Detection of fault

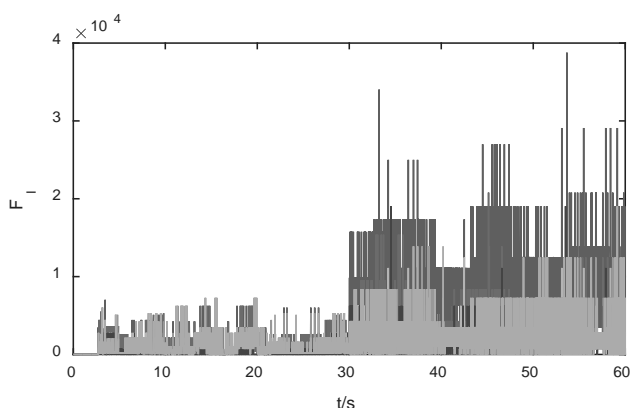

(b) Isolation of fault

Fig 5. FDI result of a $75^{\circ} / \mathrm{h}$ step fault after Quantization

\section{Solution for Quantization}

In this paper, the method of filtering is used to solve the problem of the small amplitude faults that cannot be detected. After the inertial navigation quantization output, add a low pass filter, which can attenuate over-high quantization noise:

$$
G=\frac{1}{T s+1}
$$

In the first order low pass filter, the time constant $\mathrm{T}$ is set to be 0.5 . Obtain filter parameters by experiments. In order to reduce the number of filters and the amount of computation, add the filter to the equivalent vector $p$ in GLT method, and the elements in the $p$ can be filtered because $p$ can be regarded as a linear combination of output $Q$. Therefore, the effect that the element of $Q$ is filtered is the same as that of $p$. However, $p$ is the dimension vector of $6 \times 1$, while $Q$ is the dimension of $9 \times 1$. Therefore, it can save 3 filters.

Similarly, in 30 seconds, the step fault signal is injected into the gyro of the number 1 . After filtering, based on GLT method, the curve of the fault detection decision function is shown to be obvious in 30.4 seconds, which provides the condition for fault detection, as is shown in Fig6(a).

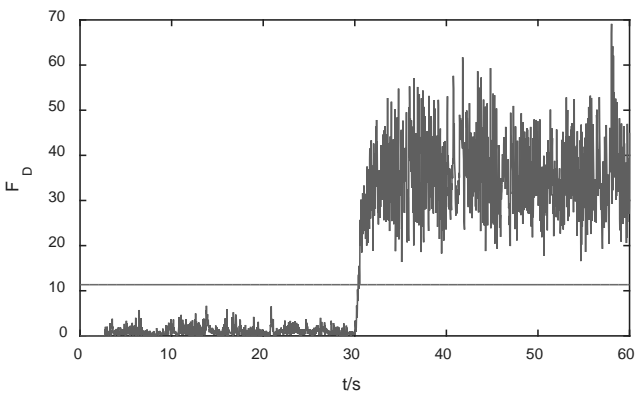

(a) Detection of fault

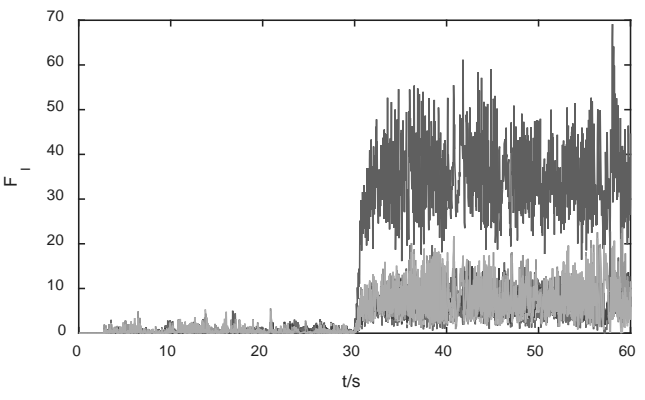

(b) Isolation of fault

Fig 6. FDI result after quantization using a low pass filter

Compared with the fault diagnosis that has not been quantitative, the fault diagnosis after the filtering should be delayed about 20 sampling periods, but it is better than fault diagnosis that has not been quantitative, for it can detect fault. Under the step signal with the amount of $50 \%$, it is almost no delay.

\section{Conclusion}

By comparing the effect of GLT method in the detection and isolation of fault diagnosis under 
three SINS before and after quantization, this paper finds that quantization results in the effects of error amplification and brings about difficulty to fault diagnosis. Finally, this paper quantifies the SINS output by a low pass filter, reduces the excessive noise, and successfully detects and isolates the small amplitude faults that cannot be detected before. However, as for the selection of the filter, we still need further studies.

\section{References}

[1] Hall S R, Paul Motyka, Eliezer Gai, etc. In-Flight Parity Vector Compensation for FDI[J]. IEEE Trans. on Aerospace and Electronic Systems, 1983. AES-19(5): 668-675

[2] Richard A M, Howard M. Redundancy management of inertial systems[J]. AIAA Paper No.73-852, 1973.7

[3] Duk-Sun Shim, Cheol-Kwan Yang. Optimal Configuration of Redundant Inertial Sensors for Navigation and FDI Performance[J]. Sensors, 2010, 10(7): 6497-6512.

[4] Gilmore J P, McKem R A. A Redundant Strapdown Inertial Reference Unit(SIRU)[J]. J.Spacecraft, Vol.9 No.1 1972.

[5] Potter J E, Suman M C. Thresholdless Redundancy Management with Arrays of Skewed Instruments[J]. Integrity in Electronic Flight Control Systems, 1977, 15(1): 15-25.

[6] Kevin C D, Eliezer Gai, James V H. Generalized Likelihood Test for FDI in Redundant Sensor Configurations [J].Guidance and Control (S1057-4932), 1978, 2(1): 9-17.

[7] Jin Hong, Zhang Hongyue. Optimal Parity Vector Sensitive to Designated Sensor Fault[J]. IEEE Transactions on Aerospace and Electronic Systems, 1999, 35(4): 1122-1128.

[8] Zhao Gang, Li Yanjun. Spacecraft Fault Diagnosis Method Based on Time Series Data Mining[J]. Journal of Spacecraft TT\&C Technology, 2010, 29(3): 1-5 (in Chinese)

[9] Duk-Sun Shim, Cheol-Kwan Yang. Geometric FDI based on SVD for Redundant Inertial Sensor Systems[J]. 2004 5th Asian Control Conference, 2004, 6: 1094- 1100.

[10]Wei Wei, Lu Zhidong, Qin Yongyuan. Soft fault detection of redundant sensor SINS[J]. Journal of Chinese Inertial Technology, 2009, 17(1): 111-116

[11]Wang Mingang, Fan Yingping, Cui Weicheng, et al. A Study of the Redudancy System Using Four Gyroscopes[J]. Journal of Projectiles, Rockets, Missiles and Guidance, 2006. 26(3): 40-42. (in Chinese)

[12]Paul G S. Analytical Modeling of Sensor Quantization in Strapdown Inertial Navigation Error Equations[J]. Journal of Guidance, Control, and Dynamics, 2002, 25(5): 833-842

[13] James C W. Competitive Evaluation of Failure Detection Algorithms for Strapdown Redundant Inertial Instruments[R]. TRW Report No. 18313-6004-RU-00 NASA.1973. 40-41 